\title{
Recent results on search for new physics at BaBar
}

\author{
Benjamin Oberhof ${ }^{1, a}$ \\ ${ }^{1}$ Laboratori Nazionali di Frascati dell'INFN, Frascati, Italy
}

\begin{abstract}
We present some recent measurements for the search of New Physics using $514 \mathrm{fb}^{-1}$ of $e^{+} e^{-}$ collisions collected with the BABAR detector at the PEP-II $e^{+} e^{-}$collider at SLAC. First we present a search for the decay $\Upsilon(1 S) \rightarrow \gamma A^{0}, A^{0} \rightarrow c \bar{c}$, where $A^{0}$ is a candidate for the CP-odd Higgs boson of the next-tominimal supersymmetric standard model. No significant signal is observed and we set $90 \%$ confidence-level upper limits on $B\left(\Upsilon(1 S) \rightarrow \gamma A^{0}\right) \times B\left(A^{0} \rightarrow c \bar{c}\right)$. We report the search for a light non-Standard Model gauge boson $Z^{\prime}$ coupling only to the second and third lepton families. Our results significantly improve current limits and further constrain the remaining region of the allowed parameter space. Finally, we present a search for a long-lived particle $L$ that is produced in $e^{+} e^{-}$annihilations and decays into two oppositely charged tracks. We do not observe a significant signal and we and set $90 \%$ confidence level upper limits on the product of the $L$ production cross section, branching fraction, and reconstruction efficiency as a function of the $L$ mass. In addition, upper limits are provided on the branching fraction $B\left(B \rightarrow X_{s} L\right)$, where $X_{s}$ is an hadronic system with strangeness -1 .
\end{abstract}

\section{Introduction}

The Standard Model (SM) of particle physics has proven to be very successful in explaining the fundamental laws of nature, and most of the theoretical results of the SM agree with the experimental data. The SM, however, cannot be quantified as a complete theory of fundamental interactions as it shows theoretical drawbacks and it is unable to explain some experimental observations. The so called "hierarchy problem" requires the fine tuning of the Higgsboson mass in order that the quadratical divergences occurring in loop corrections are systematically canceled and is deemed unnatural by many theorists. Some extensions of the SM account for this problems and naturally stabilize the hierarchy between the electroweak and the Plank scale [1]. Experimentally, astrophysical and cosmological observations indicate that a fraction of energy density in the universe is due to non-baryonic matter, usually called dark matter (DM). The nature of DM is currently unknown, and the SM does not have viable candidate for DM particles. Many models of physics beyond the SM predict the existence of a new gauge groups with gauge boson masses below $10 \mathrm{GeV}$ which can typically interact with other SM elementary particles through so-called portals [2].

We herein present three different searches for new physics candidates using data recorded by the BABAR detector at the PEP-II asymmetric-energy $e^{+} e^{-}$storage rings operated at the SLAC National Accelerator Laboratory. The data sample consists of $424 \mathrm{fb}^{-1}$ of $e^{+} e^{-}$collisions recorded at the center-of-mass $(\mathrm{CM})$ energy of the $\Upsilon(4 S)$ resonance, $27.8 \mathrm{fb}^{-1}$ of data recorded at the $\Upsilon(3 S)$ and

\footnotetext{
${ }^{\mathrm{a}} \mathrm{e}$-mail: benjamin.oberhof $@ \operatorname{lnf}$. infn.it
}

$13.6 \mathrm{fb}^{-1}$ of data collected at the $\Upsilon(2 S)$ resonance. A detailed description of the $B A B A R$ detector is given elsewhere [3].

\section{Search for a light Higgs in radiative decays of the $\Upsilon(1 S)$ with a charm tag}

An appealing extension of the Standard Model is represented by the next-to-minimal supersymmetric standard model (NMSSM) since it solves both the $\mu$ problem of the minimal supersymmetric standard model (MSSM) as well as the hierarchy problem of the SM $[4,5]$. The NMSSM has an extended Higgs sector composed of two charged, three neutral CP-even, and two neutral CP-odd bosons. The mass of the lightest NMSSM Higgs boson $A^{0}$ could be low enough to be produced in the decay of the $\Upsilon(1 S)$ [6]. The branching fractions of the $A^{0}$ to SM particles will depend on the $A^{0}$ mass and the NMSSM parameter $\tan \beta$ [7]. BABAR has already performed searches below the charm mass threshold searching for $A^{0} \rightarrow \mu^{+} \mu^{-}[8,9]$ and for $A^{0} \rightarrow g g$ or $s \bar{s}$ [10] as well as above the charm mass threshold in $A^{0} \rightarrow \tau^{+} \tau^{-}$searches [11]. None of these searches have observed a significant signal and most of the NMSSM parameter space has been ruled out. Here we report a search for the decay $\Upsilon(1 S) \rightarrow \gamma A^{0}, A^{0} \rightarrow c \bar{c}$ with $A^{0}$ masses ranging from 4.00 to $9.25 \mathrm{GeV} / \mathrm{c}^{2}$. For this search we use $13.6 \mathrm{fb}^{-1}$ of data collected at the $\Upsilon(2 S)$ resonance, corresponding to $(98.3 \pm 0.9) \times 10^{6} \Upsilon(2 S)$ mesons [12], which includes an estimated $(17.5 \pm 0.3) \times 10^{6} \Upsilon(2 S) \rightarrow$ $\pi^{+} \pi^{-} \Upsilon(1 S)$ decays [13]. The non- $\Upsilon(2 S)$ backgrounds are studied using $1.4 \mathrm{fb}^{-1}$ of "off-resonance" data collected $30 \mathrm{MeV}$ below the $\Upsilon(2 S)$ resonance. A Monte Carlo 
(MC) simulation is used to estimate the signal efficiency and optimize the search as well as for background studies. The signal decay chain $e^{+} e^{-} \rightarrow \Upsilon(2 S) \rightarrow \pi^{+} \pi^{-} \Upsilon(1 S)$, $\Upsilon(1 S) \rightarrow \gamma A^{0}, A^{0} \rightarrow c \bar{c}$ is simulated with EVTGEN [14], for $A^{0}$ masses between 4.0 and $9.0 \mathrm{GeV} / \mathrm{c}^{2}$ in $0.5 \mathrm{GeV} / \mathrm{c}^{2}$ steps and for $A^{0}$ masses of $9.2,9.3$, and $9.4 \mathrm{GeV} / \mathrm{c}^{2}$. The $A^{0}$ decay width is assumed to be $1 \mathrm{MeV}$. The hadronization of the $c \bar{c}$ system is simulated using the JETSET [15] program. The detector response is simulated with GEANT4 [16]. $\Upsilon(1 S)$ decays are tagged by the presence of a pion pair from $\Upsilon(2 S) \rightarrow \pi^{+} \pi^{-} \Upsilon(1 S)$. To tag the $A^{0} \rightarrow c \bar{c}$ decay at least one charmed meson such as a $D^{0}$, a $D^{+}$, or a $D^{*}(2010)^{+}$is required. In this analysis the $A^{0}$ is not fully reconstructed, instead the search for the $A^{0}$ is performed in the spectrum of the invariant mass of the system recoiling against the $\pi^{+} \pi^{-} \gamma$ system. Events are required to contain at least one photon candidate with $E>30$ $\mathrm{MeV}$. Each photon candidate is taken in turn to represent the signal candidate in the $\Upsilon(1 S) \rightarrow \gamma A^{0}$ decays, allowing multiple candidates per event. $D$ meson candidates are reconstructed in five channels: $D^{0} \rightarrow K^{-} \pi^{+}$, $D^{+} \rightarrow K^{-} \pi^{+} \pi^{+}, D^{0} \rightarrow K^{-} \pi^{+} \pi^{-} \pi^{+}, D^{0} \rightarrow K_{S}^{0} \pi^{+} \pi^{-}$, and $D^{*}(2010)^{+} \rightarrow \pi^{+} D^{0}$ with $D^{0} \rightarrow K^{-} \pi^{+} \pi^{0}$. Events are required to have at least one dipion candidate; $m_{R}$, the invariant mass of the system recoiling against the dipion in the $\Upsilon(2 S) \rightarrow \pi^{+} \pi^{-} \Upsilon(1 S)$, is calculated as

$$
m_{R}^{2}=M_{\Upsilon(2 S)}^{2}+m_{\pi \pi}^{2}-2 M_{\Upsilon(2 S)} E_{\pi \pi}
$$

where $M_{\Upsilon(2 S)}$ is the nominal $\Upsilon(2 S)$ mass, $m_{\pi \pi}$ and $E_{\pi \pi}$ are the measured dipion mass and energy in the center-ofmass (CM) frame. Signal candidates must satisfy $9.45<$ $m_{R}<9.47 \mathrm{GeV} / \mathrm{c}^{2}$. The mass of the $A^{0}$ candidate, $m_{X}$, is determined from the mass of the system recoiling against the dipion and photon through

$$
m_{X}=\left(P_{e+e-}-P_{\pi+\pi-}-P_{\gamma}\right)
$$

where $\mathrm{P}$ denotes four-momentum measured in the $\mathrm{CM}$ frame. Simulated background include $\Upsilon(2 S)$ and $e^{+} e^{-} \rightarrow$ $q \bar{q}$ events, where $q$ is a $u, d, s$, or $c$ quark. For $m_{X}$ greater than $7.50 \mathrm{GeV} / c^{2}$, events with low-energy photons constitute an important background source, for this reason, the analysis is divided into a low mass region, for $4.00<m_{A^{0}}<8.00 \mathrm{GeV} / c^{2}$ and a high mass region, for $7.50<m_{A^{0}}<9.25 \mathrm{GeV} / c^{2}$. The overlap of the two regions is motivated by the need to have sufficient statistical precision for the background determination in each region. We train one Boosted Decision Tree (BDT) classifier [17] taking 24 different variables as input to separate background from signal candidates for each of the five $D$ channels and for each mass region. To train the BDTs samples of simulated signal events, simulated generic $\Upsilon(2 S)$ events, and the off-resonance data are used. For each channel in the two mass intervals, we require the BDT output to exceed a value determined maximizing the quantity $S /(1.5+\sqrt{B})$ [18], where $S$ is the expected number of signal events and $B$ the expected number of background events, respectively. After selection, $9.8 \times 10^{3}$ and $7.4 \times 10^{6}$ candidates satisfy the selection criteria in the low- and high-mass regions,
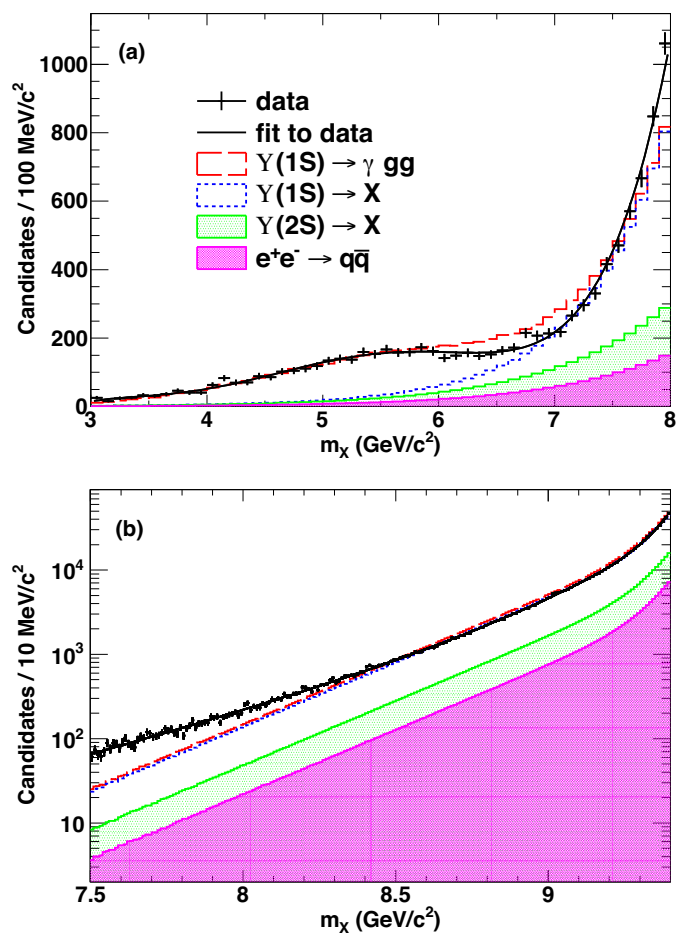

Figure 1. The $m_{X}$ distribution of signal candidates in the low(a) and high- (b) mass regions after applying all selection criteria. The points indicate the data. The solid curve shows the result of a fit to the data under a background- only hypothesis. The coloured histograms show the cumulative background contributions from $e^{+} e^{-} \rightarrow q \bar{q}$ (magenta dense-dot filled), $\Upsilon(2 S) \rightarrow X$ (green sparse-dot filled), $\Upsilon(1 S) \rightarrow X$ (blue dotted), and $\Upsilon(1 S) \rightarrow \gamma g g$ (red dashed) events.

respectively. The corresponding distributions of $m_{X}$ are shown in Fig. 1.

The backgrounds consist of $\Upsilon(1 S) \rightarrow \gamma g g$, other $\Upsilon(1 S) \rightarrow X$ decays, $\Upsilon(2 S) \rightarrow X$ decays without a dipion transition and $e^{+} e^{-} \rightarrow q \bar{q}$ events. The corresponding contributions are $35 \%, 34 \%, 15 \%$ and $16 \%$ respectively in the low-mass region and $1 \%, 66 \%, 18 \%$, and $15 \%$ respectively in the high-mass region. The contribution from $\Upsilon(1 S) \rightarrow \gamma g g$ decays reaches a maximum near $5.5 \mathrm{GeV} / c^{2}$ and decreases above $7 \mathrm{GeV} / c^{2}$. We search for the $A^{0}$ resonance as a peak in the $m_{X}$ distribution; the scan is performed by means of extended maximum likelihood fits as a function of test-mass values, denoted $m_{A}^{0}$. For these fits the shape of the signal distribution are fixed while the parameters of the background PDF, the number of signal events $N_{\text {sig }}$, and the number of background events are determined in the fit. The signal PDF is modeled with a Crystal Ball function [19]. The background PDF is modeled with a second-order polynomial. The fits are performed in steps of 10 and $2 \mathrm{MeV} / c^{2}$ in the low- and high-mass regions, respectively. We use a local fitting range of $\pm 10 \sigma_{C B}$ around 
test-mass values, where $\sigma_{C B}$ is the width of the Gaussian component of the Crystal Ball function. The $\sigma_{C B}$ parameter varies between 120 and $8 \mathrm{MeV} / c^{2}$ for values of $m_{A}^{0}$ between 4.00 and $9.25 \mathrm{GeV} / c^{2}$. In the high-mass region we exclude the interval $8.95<m_{A}^{0}<9.10 \mathrm{GeV} / c^{2}$ because of a large background from $\Upsilon(2 S) \rightarrow \gamma \chi_{b J}(1 P), \chi_{b J}(1 P) \rightarrow$ $\gamma \Upsilon(1 S)$ decays. The fitting procedure is validated with background-only pseudo-experiments; a fifth-order polynomial PDF is used to fit the data and generate the pseudoexperiments in the low-mass region, while a sum of four exponential functions and six Crystal Ball functions is used for the high-mass region. The Crystal Ball functions describe the $\Upsilon(2 S) \rightarrow \gamma \chi_{b J}(1 P)$ and $\chi_{b J}(1 P) \rightarrow \gamma \Upsilon(1 S)$ transitions while the exponential terms describe the nonresonant background. The fitting procedure is found to require a correction to $N_{\text {sig }}$ for values of $m_{A^{0}}$ near 4.00 and $9.25 \mathrm{GeV} / c^{2}$. The corrections are determined from the average signal yield found in the fits to the background-only pseudo-experiments. The maximum correction reaches 50 candidates in the high-mass region; the uncertainty of the correction is taken to be half its value. The overall efficiencies range from $4.0 \%$ to $2.6 \%$ between 4.00 and 9.25 $\mathrm{GeV} / c^{2}$. Potential bias introduced by the fitting procedure is evaluated using pseudo-experiments which use different values of $B\left(\Upsilon(1 S) \rightarrow \gamma A^{0}\right) \times B\left(A^{0} \rightarrow c \bar{c}\right)$; depending on $m_{A^{0}}$, the extracted product branching fraction is found to be up to $(4 \pm 3) \%$ higher than the value used to generate the events. The systematic uncertainties associated with the reconstruction efficiencies are dominated by the differences between data and simulation, including the BDT output modelling, $c \bar{c}$ hadronization, $D$-candidate mass resolution, dipion recoil mass and likelihood modeling, and photon reconstruction. Other systematic uncertainties are associated with the fit bias, the dipion branching fraction, the finite size of the simulated signal sample, and the $\Upsilon(2 S)$ counting. The BDT output distributions in off-resonance data and $e^{+} e^{-} \rightarrow q \bar{q}$ simulation, are slightly shifted from one another; the associated systematic uncertainty is estimated by shifting the simulated distributions so that the mean values agree with the data, and then recalculating the efficiencies. The reconstruction efficiencies decrease by $7 \%$ and $2 \%$ in the low- and high-mass regions, respectively. Uncertainty associated with $c \bar{c}$ hadronization is evaluated by comparing $D$ meson production in offresonance data and $e^{+} e^{-} \rightarrow c \bar{c}$ simulation; the difference varies from $1 \%$ to $9 \%$ for the five $D$ decay channels. We conservatively assign a global multiplicative uncertainty of $9 \%$ that includes effects due to the hadronization modeling, particle identification, tracking, $\pi^{0}$ reconstruction, and luminosity determination of the off-resonance data. The uncertainty due to the discrepancy between the reconstructed $D$ mass resolution in data and simulation is estimated by gaussian smearing of the $D$ mass input in simulation to match the data and measuring the difference in the reconstruction efficiency. Further corrections to account for data and simulation differences in reconstruction efficiencies are estimated with similar methods. Corrections are applied to account for the dipion recoil mass recon- struction, the dipion likelihood modeling, and the photon reconstruction [20].

The highest observed local significance in the lowmass region is $2.3 \sigma$, at $4.145 \mathrm{GeV} / c^{2}$, and $2.0 \sigma$ at 8.411 $\mathrm{GeV} / \mathrm{c}^{2}$ in the high-mass region. Such fluctuations occur in $54 \%$ and $80 \%$ of pseudo-experiments, respectively, hence we conclude that our data are consistent with the background-only hypothesis [21]. Bayesian upper limits on the product branching fraction $B\left(\Upsilon(1 S) \rightarrow \gamma A^{0}\right) \times$ $B\left(A^{0} \rightarrow c \bar{c}\right)$ at $90 \%$ confidence level (C.L.) are determined assuming a uniform prior, with the constraint that the product branching fraction be greater than zero. The distribution of the likelihood function for $N_{\text {sig }}$ is assumed to be gaussian with a width equal to the total uncertainty on $N_{\text {sig }}$. The upper limits obtained from the low-mass region are combined with those from the high-mass region to define a continuous spectrum. The results are shown in Fig. 2.

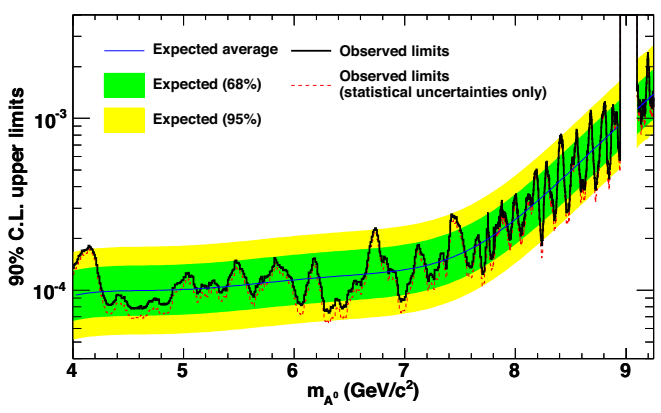

Figure 2. $90 \% \mathrm{CL}$ upper limits for product branching fraction $\mathcal{B}\left(\Upsilon(1 S) \rightarrow \gamma A^{0}\right) \times \mathcal{B}\left(A^{0} \rightarrow c \bar{c}\right)$ including all uncertainties (thick line) and statistical uncertainties only (thin dashed line). The inner and outer bands represent the $68 \%$ and $95 \%$ expected upper limits. The bands are calculated using all uncertainties. The solid line in the center of the inner band is the expected value of the upper limit.

\section{Search for a muonic dark force}

In the SM particles and interactions are insufficient to explain cosmological and astrophysical observations of dark matter. A possible scenario is represented by new hidden sectors that are only feebly coupled to the SM. In the simplest case of a hidden U(1) gauge interaction, such a sector contains it's own gauge bosons $Z^{\prime}$ and SM fields may directly couple to the $Z^{\prime}$, or alternatively the $Z^{\prime}$ boson may mix with the SM hypercharge boson [22]. In the latter case, the $Z^{\prime}$ couplings are proportional to the SM gauge couplings; however, due to large couplings to electrons and light-flavor quarks, such scenarios are strongly constrained by existing searches [23]. If SM fields are directly charged under the dark force instead, the $Z^{\prime}$ may interact preferentially with heavy-flavor leptons, greatly 
reducing the sensitivity of current searches. Such interactions could account for the experimentally measured value of the muon anomalous magnetic dipole moment [24], as well as the discrepancy in the proton radius extracted from measurements of the Lamb shift in muonic hydrogen compared to observations in non-muonic atoms $[25,26]$. In the following we report a search for dark bosons $Z^{\prime}$ with vector couplings only to the second and third generations of leptons [27] in $e^{+} e^{-} \rightarrow \mu^{+} \mu^{-} Z^{\prime}, Z^{\prime} \rightarrow \mu^{+} \mu^{-}$.

For this search we use the full $\Upsilon(4 S)$ data sample plus about $28 \mathrm{fb}^{-1}$ data at $\Upsilon(3 S), 14 \mathrm{fb}^{-1}$ data at $\Upsilon(2 S)$, and 48 $\mathrm{fb}^{-1}$ off-resonance data. About $5 \%$ of the data set is used to validate and optimize the analysis method, the rest of the data was only examined after finish finalizing the analysis method. For the background study we use MC samples. Signal MC events are generated using MadGraph 5 [28] and hadronized in Pythia 6 [29] for $Z^{\prime}$ mass hypotheses from the dimuon mass threshold to $10.3 \mathrm{GeV} / \mathrm{c}^{2}$. Background samples include the direct processes of $e^{+} e^{-} \rightarrow$ $\mu^{+} \mu^{-} \mu^{+} \mu^{-}$generated with Diag36 [30], which includes the full set of the lowest order diagrams. The events of the process of $e^{+} e^{-} \rightarrow e^{+} e^{-}(\gamma)$ are generated using BHWIDE [31] while of $e^{+} e^{-} \rightarrow \mu^{+} \mu^{-}(\gamma)$ and $e^{+} e^{-} \rightarrow \tau^{+} \tau^{-}(\gamma)$ are generated using KK2 $\mathrm{f}$ [32]. The off-resonance data samples, $e^{+} e^{-} \rightarrow q \bar{q}(q=u, d, s, c)$, are simulated using JETSET. The events processes of $e^{+} e^{-} \rightarrow \psi(2 S) \gamma$ followed by $\psi(2 S) \rightarrow \pi^{+} \pi^{-} J / \psi$ and $J / \psi \rightarrow \mu^{+} \mu^{-}$were generated using EVTGEN with appropriate phase-space model. Finally the detector acceptance and reconstruction efficiency are determined using GEANT4.

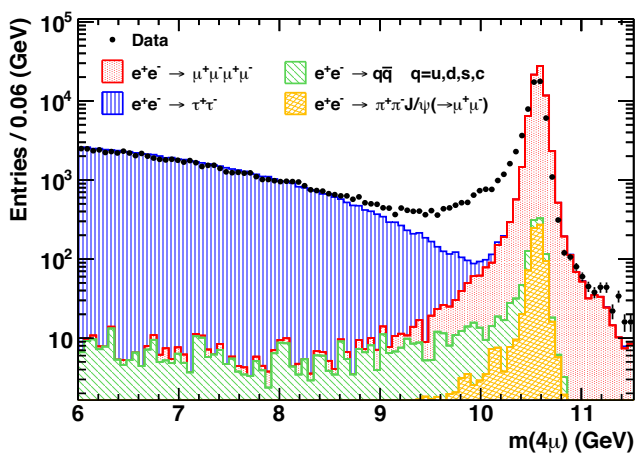

Figure 3. The distribution of the four-muon invariant mass, $m_{4 \mu}$, for data taken at the $\Upsilon(4 S)$ peak together with Monte Carlo predictions of various processes normalized to data luminosity. The $e^{+} e^{-} \rightarrow \mu^{+} \mu^{-} \mu^{+} \mu^{-}$MC does not include ISR corrections.

We select events with exactly two pairs of oppositely charged tracks, consistent with the $e^{+} e^{-} \rightarrow \mu^{+} \mu^{-} Z^{\prime}, Z^{\prime} \rightarrow$ $\mu^{+} \mu^{-}$final state. The muons are identified by multivariate particle identification algorithms for each track. We require the sum of energies of the deposits in the electromagnetic calorimeter that are not associated to any track to be less than $200 \mathrm{MeV}$. We reject events coming from the $\Upsilon(3 S)$ and $\Upsilon(2 S)$, where $\Upsilon(2 S, 3 S) \rightarrow \pi^{+} \pi^{-} \Upsilon(1 S)$,
$\Upsilon(1 S) \rightarrow \mu^{+} \mu^{-}$decays if the dimuon combination lies within $100 \mathrm{MeV} / c^{2}$ of the $\Upsilon(1 S)$ mass. The distribution of the four-muon invariant mass after all selections is shown in Fig. 3. The lower part of the four-muon invariant mass spectrum, $m_{4 \mu}<9 \mathrm{GeV} / c^{2}$, is well reproduced by the Monte Carlo simulation while the MC simulation overestimates the full energy peak by about $30 \%$ and fails to reproduce the radiative tail. This, however, is expected because the Diag36 simulation does not simulate the initial state radiation (ISR). We select $e^{+} e^{-} \rightarrow \mu^{+} \mu^{-} \mu^{+} \mu^{-}$events by requiring a four-muon invariant mass distribution within 500 $\mathrm{MeV} / \mathrm{c}^{2}$ of the nominal center-of-mass energy. We also require the tracks to originate from the interaction point to within its uncertainty and constraining the center-of-mass energy of the system to be within the beam energy spread. We do not attempt to select a single $Z^{\prime} \rightarrow \mu^{+} \mu^{-}$candidate per event, but instead consider all possible combinations. The most important contribution on the invariant mass peak besides the QED process $e^{+} e^{-} \rightarrow \mu^{+} \mu^{-} \mu^{+} \mu^{-}$ comes from $\Upsilon(2 S) \rightarrow \pi^{+} \pi^{-} J / \psi, J / \psi \rightarrow \mu^{+} \mu^{-}$as can be seen in Fig. 3. We extract the signal yield by a series of unbinned likelihood fits to the spectrum of the reduced dimuon mass $m_{R}$, defined as $m_{R}=\sqrt{m_{\mu^{+} \mu^{-}}^{2}-4 m_{\mu}^{2}}$, within the range of $0.212<m_{R}<10 \mathrm{GeV} c^{2}$ and $0.212<m_{R}<9$ $\mathrm{GeV} c^{2}$ for the $\Upsilon(4 S)$ resonance data and $\Upsilon(2 S)$ and $\Upsilon(3 S)$ resonances data, respectively. We exclude a region of \pm 30 $\mathrm{MeV} c^{2}$ around the nominal known $J / \psi$ mass. We probe a total of 2219 mass hypothesis. The signal efficiency at low masses is about $35 \%$ and it rises to about $50 \%$ around $m_{R}=6-7 \mathrm{GeV} / c^{2}$ to drop again at higher values of the reduced dimuon mass. The signal efficiencies include a correction factor of 0.82 , which accounts for the impact of ISR not included in the simulation, as well as differences between data and simulation in trigger efficiency, charged particle identification, and track and photon reconstruction efficiencies. This correction factor is obtained from the ratio of the $m_{R}$ distribution in simulated $e^{+} e^{-} \rightarrow \mu^{+} \mu^{-} \mu^{+} \mu^{-}$ events to the observed distribution in the mass region 1-9 $\mathrm{GeV} / c^{2}$. We also assign a systematic uncertainty of $5 \%$ to cover the small variations between the uncertainties on the $e^{+} e^{-} \rightarrow \mu^{+} \mu^{-} \mu^{+} \mu^{-}$and data taking period. We calculate the ISR contribution based on the quasi real electron approximation [38].

The cross section of $e^{+} e^{-} \rightarrow \mu^{+} \mu^{-} Z^{\prime}, Z^{\prime} \rightarrow \mu^{+} \mu^{-}$is extracted as a function of $Z^{\prime}$ mass. The gray band indicates the excluded region. We find the largest local significance is $4.3 \sigma$ around $Z^{\prime}$ mass of $0.82 \mathrm{GeV} / c^{2}$ that is corresponding to the global significance of $1.6 \sigma$ and it is consistent with the null-hypothesis [33]. We also derive 90\% confidence level (CL) Bayesian upper limit on the cross section of $e^{+} e^{-} \rightarrow \mu^{+} \mu^{-} Z^{\prime}, Z^{\prime} \rightarrow \mu^{+} \mu^{-}$as shown in Fig. 4. We consider all uncertainties to be uncorrelated except for the uncertainties of the luminosity and efficiency. We finally extract the corresponding $90 \%$ CL on the coupling parameter $g^{\prime}$ by assuming the equal magnitude vector couplings muons, taus and the corresponding neutrinos together with the existing limits from Borexino and neutrino experiments as shown in Fig. 5. We set down to $7 \times 10^{-4}$ near the dimuon threshold. 


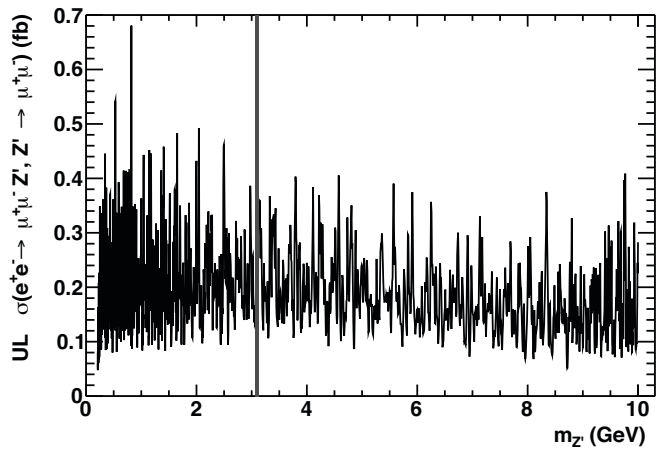

Figure 4. The $90 \% \mathrm{CL}$ upper limits on the cross-section $\sigma\left(e^{+} e^{-} \rightarrow \mu^{+} \mu^{-} Z^{\prime}, Z^{\prime} \rightarrow \mu^{+} \mu^{-}\right)$as a function of the $Z^{\prime}$ mass. The dark gray band indicates the region excluded from the analysis.

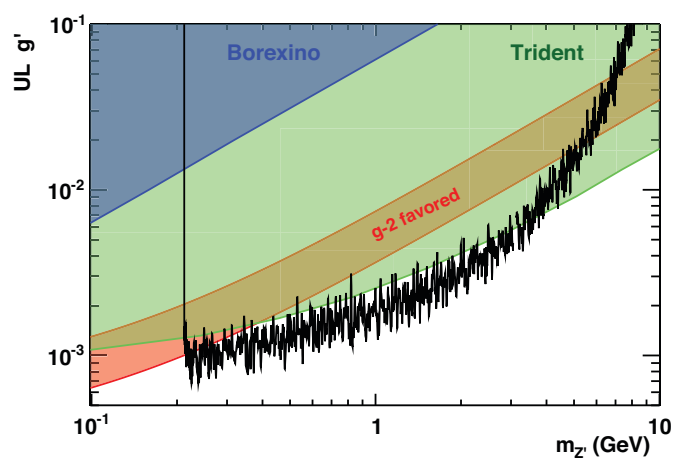

Figure 5. The $90 \%$ CL upper limits on the new gauge coupling $g^{\prime}$ as a function of the $Z^{\prime}$ mass, together with the constraints derived from the production of a $\mu^{+} \mu^{-}$pairs in $v_{\mu}$ scattering ("Trident" production) [34]. The region consistent with the discrepancy between the calculated and measured anomalous magnetic moment of the muon within $2 \sigma$ is shaded in red.

\section{Search for long lived particles}

Anomalous astrophysical observations [35]-[37] have generated some interest in $\mathrm{GeV}$-scale hidden-sector long-lived states [38]. BABAR offers an ideal laboratory to search for such a particle in the $\mathrm{O}\left(1 \mathrm{GeV} / c^{2}\right)$ mass range. We thus search for a neutral, long-lived particle $L$, which decays into one of the following final states $f=e^{+} e^{-}, \mu^{+} \mu^{-}$, $e^{ \pm} \mu^{\mp}, \pi^{+} \pi^{-}, K^{+} K^{-}$, or $K^{ \pm} \pi^{\mp}$. The signature for such a decay is a displaced vertex consistent with a two-body decay. The search is performed by fitting the $L$-candidate mass distribution. We provide "model-independent" limits on the product $\sigma\left(e^{+} e^{-} \rightarrow L X\right)(L \rightarrow f) \epsilon(f)$ of the inclusive production cross section $\sigma\left(e^{+} e^{-} \rightarrow L X\right)$, the branching fraction $B(L \rightarrow f)$, and efficiency $\epsilon(f)$ for each of the two-body final states $f$, where $X$ is any set of particles. Detailed tables for efficiency as a function of $L$ mass $m$, transverse momentum, and proper decay distance $c \tau$ are given in [39]. We also provide "modeldependent" limits on the branching fraction for the decay $B \rightarrow X_{s} L$, where $X_{s}$ is an hadronic system with strangeness -1 . To determine signal mass resolution and reconstruction efficiency we use MC simulations; signal events are produced with the EVTGEN generator, assuming the $L$ spin to be zero, in two different ways: for the "model-independent" search the $L$ is produced at 11 different masses, $m_{M C}=0.5,1,2,3,4,5,6,7,8,9$, and 9.5 $\mathrm{GeV} / c^{2}$; for $m_{M C} \leq 4 \mathrm{GeV} / c^{2}$, the $L$ is created in the process $e^{+} e^{-} \rightarrow B \bar{B}$, with one $B$ meson decaying to $L+N \pi$ $(N=1,2$, or 3$)$ and the other $B$ meson decaying generically. while at higher masses the production process is assumed to be $\Upsilon(4 S) \rightarrow L+N \pi$. The production processes are chosen in order to populate properly the phase space and do not reflect any preferred production hypothesis; in both regions, the $L$ is produced uniformly throughout the available phase space, with an average transverse decay distance of $20 \mathrm{~cm}$. Efficiencies for other decay lengths are obtained by reweighting the events. For the "modeldependent" presentation we generate $B \rightarrow X_{S} L$ decays for the seven mass values $m_{M C}=0.5,1,2,3,3.5,4$, and 4.5 $\mathrm{GeV} / c^{2}$. The composition of the $X_{s}$ is taken to be $10 \%$ $K, 25 \% K^{*}(892)$, and $65 \% K^{*}(1680)$, with the high-mass tail of the $X_{S}$ spectrum suppressed by phase-space limitations. The other B meson in the event decays generically. In addition to the signal MC samples, we use the following background MC samples in order to optimize the event selection criteria and study the signal extraction method: $e^{+} e^{-} \rightarrow B \bar{B}$ (produced with EVTGEN), $\tau^{+} \tau^{-}, \mu^{+} \mu^{-}$(KK2f), $e^{+} e^{-}$(BHWIDE), and $q \bar{q}$ events (JETSET), where $q$ is a $u$, $d, s$, or $c$ quark. The detector response is simulated with GEANT4.

Each track must satisfy $d_{0} / \sigma_{d_{0}}>3$, where $d_{0}$ is the transverse distance of closest approach of the track to the $e^{+} e^{-}$interaction point (IP), and $\sigma_{d_{0}}$ is its uncertainty. The two tracks have to originate from a common vertex, and the $\chi^{2}$ value of the fit is required to be smaller than 10 for one degree of freedom. We require the distance $r$ between the IP and the vertex in the transverse plane to be in the range $1<r<50 \mathrm{~cm}$, and the uncertainty on $r$ is required to satisfy $\sigma_{r}<0.2 \mathrm{~cm}$. We also require the angle $\alpha$ between $\vec{r}$ and the $L$-candidate reconstructed transverse momentum vector to be $\alpha<0.01 \mathrm{rad}$. The uncertainty $\sigma_{m}$ on the measured $L$-candidate mass m must be less than $0.2 \mathrm{GeV} / c^{2}$. The $L$ candidate is discarded if either of the tracks has associated hits in the detector between the IP and the vertex, or if the vertex is within the material of the beampipe wall, or the detector bulk material. Depending on the specific final state, the candidates must satisfy the following invariant-mass criteria: $m_{e^{+} e^{-}}>0.44 \mathrm{GeV} / c^{2}$, $m_{\mu^{+} \mu^{-}}<0.37 \mathrm{GeV} / c^{2}$ or $m_{\mu^{+} \mu^{-}}>0.5 \mathrm{GeV} / c^{2}, m_{e^{ \pm} \mu^{\mp}}>0.48$ $\mathrm{GeV} / c^{2}, m_{\pi^{+} \pi^{-}}>0.86 \mathrm{GeV} / c^{2}, m_{K^{+} K^{-}}>1.35 \mathrm{GeV} / c^{2}$, and $m_{K^{ \pm} \pi^{\mp}}>1.05 \mathrm{GeV} / c^{2}$. These criteria reject most background from $K_{S}^{0} \rightarrow \pi^{+} \pi^{-}$and $\Lambda \rightarrow p \pi^{-}$decays. In addition, except for the $\mu^{+} \mu^{-}$mode, they exclude low-mass regions in which the mass distributions of background MC events are not smooth, and therefore incompatible with the 
background description method outlined below. We require at least one of the tracks of $L \rightarrow \mu^{+} \mu^{-}$candidates with $m \geq 8 \mathrm{GeV} / c^{2}$ to have an SVT hit. This rejects candidates that decay into $\mu^{+} \mu^{-}$within the material of the finalfocusing magnets, and thus have poor mass resolution. These selection criteria are found to yield near-optimal signal sensitivity given the broad range of $m$ and $r$ values of this search. For each decay mode, we determine the full efficiency $\epsilon$ for different values of $m_{M C}, c \tau$, and $p_{T}$, including the impact of detector acceptance, trigger, reconstruction, and selection criteria. The dominant source of background consists of hadronic events with high track multiplicity, where large- $d_{0}$ tracks originate mostly from $K_{S}^{0}, \Lambda, K^{ \pm}$, and $\pi^{ \pm}$decays, as well as particle interactions with detector material. Random overlaps of such tracks comprise the majority of the background candidates.

We extract the signal yield for each final state as a function of $L$ mass with unbinned extended maximumlikelihood fits of the $m$ distribution. The procedure is based on the fact that signal MC events peak in $\mathrm{m}$ while the background distribution varies slowly. The fit probability density functions (PDFs) for signal and background are constructed separately for each mode and each data sample. We scan the data in search of an $L$ signal, varying $m_{0}$ in steps of $2 \mathrm{MeV} / c^{2}$. At each scan point, we fit the data in the full mass range using the PDF $n_{S} P_{S}+n_{B} P_{B}$, where the signal and background yields $n_{S}$ and $n_{B}$ are determined in the fit. The statistical significance $S=\operatorname{sign}(n) 2 \log \left(\mathcal{L}_{S} / \mathcal{L}_{B}\right)$, where $\mathcal{L}_{S}$ is the maximum likelihood for $n_{s}$ signal events over the background yield, and $\mathcal{L}_{B}$ is the likelihood for $n_{S}=0$, is calculated for each scan point. We find two scan points with a significance $S$ greater than 3 , both in the $\mu^{+} \mu^{-}$mode in the $\Upsilon(4 S)+$ offresonance sample. The highest significance is $S=4.7$, with a signal yield of 13 events at the low-mass threshold of $m_{0}=0.212 \mathrm{GeV} / c^{2}$. The second-highest significance of $S=4.2$ occurs at $m_{0}=1.24 \mathrm{GeV} / c^{2}$, corresponding to a signal yield of 10 events. To obtain the p-values for these significances, we perform a large number of pseudo experiments on $m_{\mu^{+} \mu^{-}}$spectra generated according to the background PDF, obtained from the data. We find that the probability for $S \geq 4.7(4.2)$ anywhere in the $\mu^{+} \mu^{-}$spectrum with $m_{\mu^{+} \mu^{-}}<0.37 \mathrm{GeV} / c^{2}\left(m_{\mu^{+} \mu^{-}}>0.5 \mathrm{GeV} / c^{2}\right)$ is $4 \times 10^{-4}\left(8 \times 10^{-3}\right)$. Further study provides strong indication for material interaction background in the 0.212 $\mathrm{GeV} / c^{2}$ region. Specifically, most of the $34 \mu^{+} \mu^{-}$vertices with $m_{\mu+\mu_{-}}<0.215 \mathrm{GeV} / c^{2}$ occur inside or at the edge of detector-material regions, including 10 of the vertices that also pass the $e^{+} e^{-}$selection criteria and 10 that pass the $\pi^{+} \pi^{-}$criteria. Thus, the peak is consistent with misidentified photon conversions and hadronic interactions close to the mass threshold. We conclude that no significant signal is observed.

Systematic uncertainties on the signal yields are calculated for each scan fit separately. The dominant uncertainty is due to the background PDF; this uncertainty is a few signal events on average, and generally decreases with mass. The uncertainty due to the signal mass resolution is evaluated by comparing the mass pull distributions of $K_{S}^{0}$

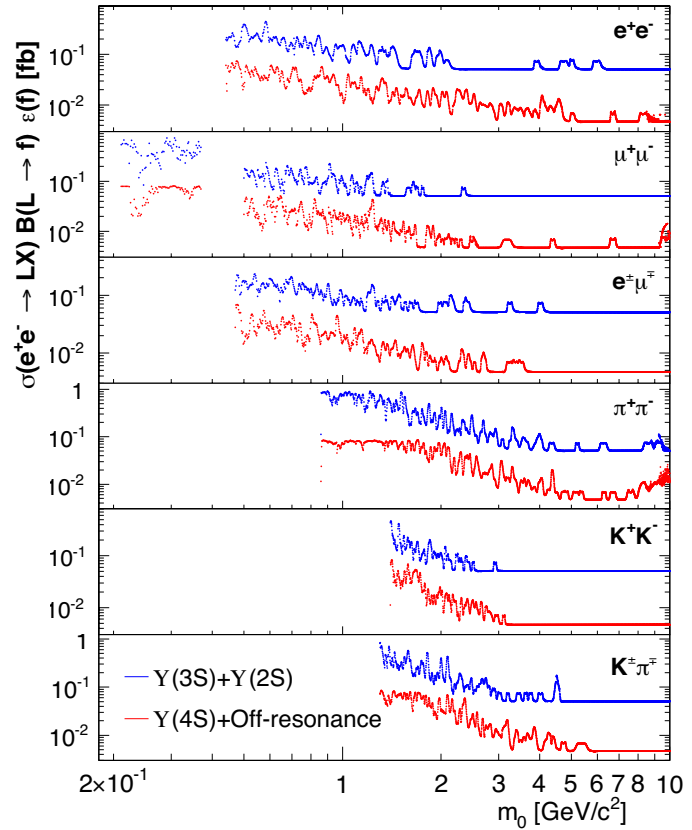

Figure 6. The $90 \%$ confidence level upper limits on $\sigma\left(e^{+} e^{-} \rightarrow\right.$ $L X) \mathcal{B}(L \rightarrow f) \epsilon(f)$ as a function of $L$ mass for the $\Upsilon(4 S)+$ offresonance sample (red lower points) and for the $\Upsilon(3 S)+\Upsilon(2 S)$ sample (blue upper points). The limits include the systematic uncertainties.

mesons in data and $\mathrm{MC}$, whose widths differ by $5 \%$. A conservative uncertainty of $2 \%$ on the signal reconstruction efficiencies is estimated from the $K_{S}^{0}$ reconstruction efficiency in data and MC. Smaller uncertainties on the efficiency, of up to $1 \%$, arise from particle identification, and signal MC statistics.

The likelihood $\mathcal{L}_{S}$ which is a nearly normal function of the signal yield, is convolved with a Gaussian representing the systematic uncertainties in $n_{S}$, to obtain the modified likelihood function $\mathcal{L}_{S}^{\prime}$. The $90 \%$ confidence level upper limit $U_{S}$ on the signal yield is calculated from $\int_{0}^{U_{S}} \mathcal{L}_{S}^{\prime} d n_{S} / \int_{0}^{\text {inf }} \mathcal{L}_{S}^{\prime} d n_{S}=0.9$. Dividing $U_{S}$ by the luminosity yields an upper limit on the product $\sigma\left(e^{+} e^{-} \rightarrow\right.$ $L X) B(L \rightarrow f) \epsilon(f)$. This limit is shown for each final state as a function of $m_{0}$ in Fig. 6 [39]. Determining the efficiency from the $B \rightarrow X_{s} L$ signal MC sample, we obtain upper limits on the product of branching fractions $B\left(B \rightarrow X_{s} L\right) B(L \rightarrow f)$ for each of the final states $f$. These limits are shown in Fig. 7.

\section{Conclusion}

In summary, we search for a resonance in radiative decays of the $\Upsilon(1 S)$ with a charm tag. We do not observe a signif- 


\section{References}

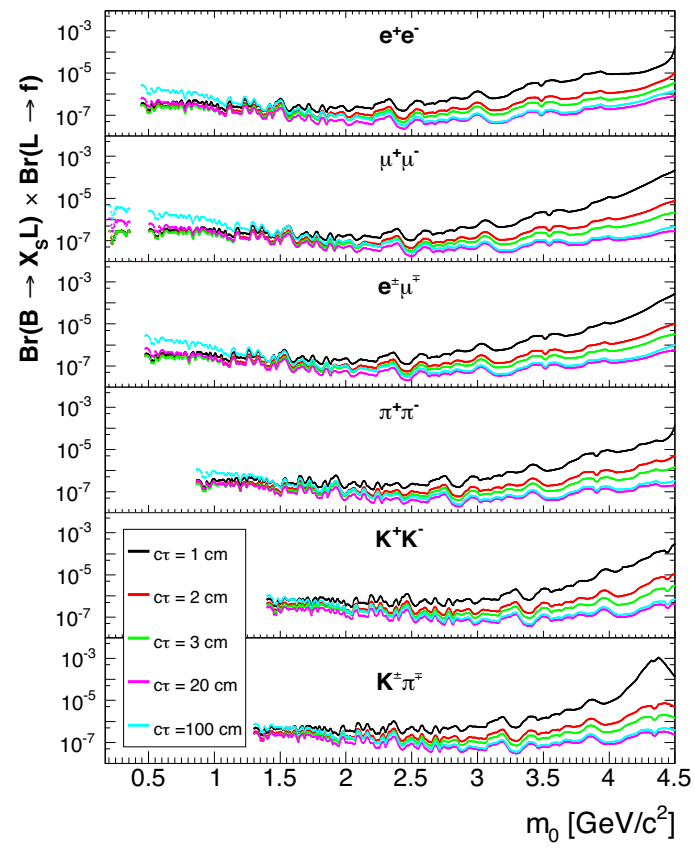

Figure 7. Implications of the results for Higgs-portal scenarios, showing the $90 \%$ confidence level upper limits on the product of branching fractions $\mathcal{B}(B \rightarrow X s L) B(L \rightarrow f)$ as a function of $L$ mass for each final state $f$ and for different values of $c \tau$. The limits include all systematic uncertainties.

icant signal and set upper limits on the product branching fraction $B\left(\Upsilon(1 S) \rightarrow \gamma A^{0}\right) \times B\left(A^{0} \rightarrow c \bar{c}\right)$ ranging from $7.4 \times 10^{-5}$ to $2.4 \times 10^{-3}$ for $A^{0}$ masses from 4.00 to 9.25 $\mathrm{GeV} / c^{2}$, excluding masses from 8.95 to $9.10 \mathrm{GeV} / c^{2}$ because of irreducible background from $\Upsilon(2 S) \rightarrow \gamma \chi_{b J}(1 P)$, $\chi_{b J}(1 P) \rightarrow \gamma \Upsilon(1 S)$ decays [21]. These results will further constrain the NMSSM parameter space.

We have performed the first direct measurement of $Z^{\prime}$ production from the decay of $e^{+} e^{-} \rightarrow \mu^{+} \mu^{-} Z^{\prime}, Z^{\prime} \rightarrow \mu^{+} \mu^{-}$. No significant signal is observed for $Z^{\prime}$ masses in the range of $0.212-10 \mathrm{GeV} / c^{2}$. We set limits on the coupling parameters $g^{\prime}$ down to $7 \times 10^{-4}$. We set a strongest bounds for many parameter space below $3 \mathrm{GeV}$. We exclude most of the remaining parameter space preferred by the discrepancy between the calculated and measured anomalous magnetic moment of the muon above the dimuon threshold [33].

We have performed a search for long-lived particles $L$ produced in $e^{+} e^{-}$collisions. No signal is observed, and upper limits on $\sigma\left(e+e^{-} \rightarrow L X\right) \mathcal{B}(L \rightarrow f) \epsilon(f)$ and on $\mathcal{B}(B \rightarrow X L) \mathcal{B}(L \rightarrow f)$ are set at $90 \%$ confidence level for six two-body final states $f$. We provide detailed efficiency tables to enable application of our results to any specific model [39].
[1] E. Witten, Phys. Lett. B 105, 267 (1981).

[2] R. Essig et al., arXiv:1311.0029 [hep-ph].

[3] B. Aubert et al. (BABAR Collaboration), Nucl. Instrum. Meth. A 479, 1 (2002); B. Aubert et al. (BABAR Collaboration), Nucl. Instrum. Meth. A 729, 615 (2013).

[4] R. Dermisek, J. F. Gunion, and B. McElrath, Phys. Rev. D 76, 051105 (2007).

[5] M. Maniatis, Int. J. Mod. Phys. A 25, 3505 (2010).

[6] F. Wilczek, Phys. Rev. Lett. 39, 1304 (1977).

[7] R. Dermisek and J. F. Gunion, Phys. Rev. D 81, 075003 (2010).

[8] B. Aubert et al. (BABAR Collaboration), Phys. Rev. Lett. 103, 081803 (2009).

[9] J. P. Lees et al. (BABAR Collaboration), Phys. Rev. D 87, 031102 (2013).

[10] J. P. Lees et al. (BABAR Collaboration), Phys. Rev. D 88, 031701 (2013).

[11] J. P. Lees et al. (BABAR Collaboration), Phys. Rev. D 88, 071102 (2013).

[12] J. P. Lees et al. (BABAR Collaboration), Nucl. Instr. Methods Phys. Res. Sect. A 726, 203 (2013).

[13] K. A. Olive et al. (Particle Data Group), Chin. Phys. C 38, 090001 (2014).

[14] D. J. Lange, Nucl. Instr. Methods Phys. Res. Sect. A 462, 152 (2001).

[15] T. Sjöstrand, Comp. Phys. Comm. 82, 74 (1994).

[16] S. Agostinelli et al., (GEANT4 Collaboration), Nucl. Instr. Methods Phys. Res. Sect. A 506, 250 (2003).

[17] A. Höcker et al., PoS ACAT, 040, (2007), physics/0703039.

[18] G. Punzi, preprint physics/0308063 (2003).

[19] M. J. Oreglia, Ph.D. Thesis, report SLAC-R-236 (1980), Appendix D.

[20] P. del Amo Sanchez et al. (BABAR Collaboration), Phys. Rev. Lett. 107, 021804 (2011).

[21] J. P. Lees et al. (BABAR Collaboration) Phys. Rev. D 91, 071102 (2015).

[22] B. Holdom, Phys. Lett. B 166, 196 (1986).

[23] A. Anastasi et al. [KLOE-2 Collaboration], Phys. Lett. B 757, 356 (2016).

[24] M. Pospelov, Phys. Rev. D 80, 095002 (2009).

[25] V. Barger, C. W. Chiang, W. Y. Keung and D. Marfatia, Phys. Rev. Lett. 106, 153001 (2011).

[26] D. Tucker-Smith and I. Yavin, Phys. Rev. D 83, 101702 (2011).

[27] X. G. He, G. C. Joshi, H. Lew and R. R. Volkas, Phys. Rev. D 43, 22 (1991).

[28] J. Alwall et al., JHEP 1407, 079 (2014).

[29] T. Sjostrand, S. Mrenna, and P. Z. Skands, JHEP 0605, 026 (2006).

[30] F. A. Berends, P. H. Daverveld and R. Kleiss, Nucl. Phys. B 253, 441 (1985).

[31] S. Jadach, W. Placzek, and B. F. L. Ward, Phys. Lett. B 390, 298 (1997). 
[32] S. Jadach, B. F. L. Ward, and Z. Was, Phys. Rev. D 63, 113009 (2001).

[33] J. P. Lees et al. (BABAR Collaboration), Phys. Rev. D 94, 011102 (2016).

[34] A. Kamada and H. B. Yu, Phys. Rev. D 92, 113004, (2015).

[35] M. Aguilar et al. [AMS Collaboration], Phys. Rev. Lett. 110, 141102 (2013).
[36] O. Adriani et al. [PAMELA Collaboration], Nature 458, 607 (2009).

[37] M. Ackermann et al. [Fermi LAT Collaboration], Phys. Rev. Lett. 108, 011103 (2012).

[38] B. Batell, D. McKeen and M. Pospelov, Phys. Rev. Lett. 107, 011803 (2011).

[39] J. P. Lees et al. (BABAR Collaboration), Phys. Rev. Lett. 114, 171801 (2015). 